\title{
Drugged driving: How much is too much?
}

\author{
Part II: Screening for cannabis-use \\ is complex
}

$\mathrm{D}$

etecting impairment from cannabis is not as straightforward as detecting alcohol because of the complex nature of the drug and lack of conclusive research about the dose-effect of cannabis.

The widely used breathalyzer test can accurately detect alcohol impairment using a breath sample because the amount of alcohol in exhaled air closely relates to the amount of alcohol in the blood, says Dr. Robert Mann, a senior scientist at the Centre for Addiction and Mental Health (CAMH). "It correspondingly increases as alcohol is first absorbed into the blood and subsequently decreases as alcohol is metabolized and removed from the blood."

But what makes breathalyzer screening viable is the substantial research showing that breath alcohol level is associated with impairment and the equally conclusive research that underpins the $0.08 \%$ blood alcohol concentration limit. That level of research isn't yet available for cannabis, argues Dr. Bernard Le Foll, head of Translational Addiction Research Laboratory at CAMH.

Cannabis' active ingredient, Delta9-tetrahydrocannabinol (THC), is very lipophilic so it goes to areas in the body full of lipids, like fat or the brain, says Le Foll. "This means what we get in the blood is just an approximation of what may be in the brain."

Although he says scientists have some idea of the relation between the blood and brain levels of THC, what is less clear is how this relates to the ability to drive. "The assumption is that it is parallel to alcohol - the more intoxicated a person, the more impairment. The reality is that we don't have such solid evidence as with alcohol of this clear relationship."

Although a number of epidemiologic studies do show that a person is more likely to be in a motor vehicle

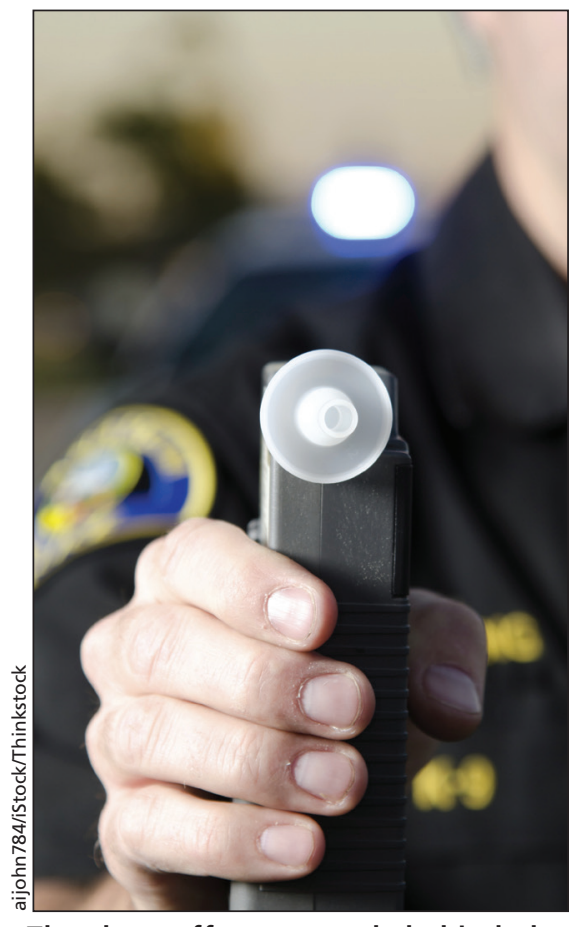

The dose-effect research behind the breathalyzer roadside alcohol test is largely lacking when it comes to cannabisuse tests.

collision while driving after using cannabis than not, Mann agrees that we don't know how this equates to the amount of THC consumed. "It's difficult to establish a dose-effect relationship ... researchers are doing work to establish something comparable to the per se level like we have for alcohol."

A 2014 review in Injury Epidemiology looking at problems in developing legal THC limits pointed to the large variability depending on the method of consumption and testing, as well as the frequency of use.

"Frequent users will reach higher levels, and cannabis will be detected longer," says Mann, citing an April 2014 study in Clinical Chemistry. According to Statistics Canada, nearly 2 million Canadians had a recurrent pattern of cannabis use in 2012, and 11 million had tried it at least once.

A further complication is the heightened danger when drivers use both alcohol and cannabis, says Le Foll. According to the Canadian Centre on Substance Abuse, $15 \%$ of fatally injured drivers in 2008 tested positive for both alcohol and drugs.

"There is some evidence that the risk of accident is much more elevated during the co-use of alcohol and cannabis, and that complicates the ability to set up a clear cut-off," says Le Foll. "Should we have a different cut-off when alcohol and cannabis are combined?"

These questions and difficulties have not stopped some jurisdictions from setting legal limits. Seven US states and 16 European countries have set limits for marijuana use enforced through chemical screening tests using driver's blood, urine or saliva.

Le Foll says that saliva testing, which can be done at the roadside, is the most effective, least invasive and simplest method. The technology is available, but the dose-response data are not. "We don't yet know much on the relationship between cannabinoid levels in saliva and the degree of impairment. There have been some studies but not so many. It has not been fully validated."

Ultimately, La Foll says the decision is in the hands of policy-makers. "Sometimes you make decisions based on what you have, and even if the data are not fully validated, you make a decision that you think is best to reduce impact on society."

Professor Robert Solomon, a law professor at Western University says using saliva to screen for cannabis may play a vital role in deterring drugimpaired driving. "I want laws that deter you, not punish you after you kill my kid. The sentence is the tail end of the dog."

In Canada, implementing roadside tests for cannabis would require the law to change, which is the subject of the last article in this series. - Dane Wanniarachige, London, Ont.

CMAJ 2015. DOI:10.1503/cmaj.109-5012 